Témoigner Témoigner. Entre histoire et mémoire

Getuigen Revue pluridisciplinaire de la Fondation Auschwitz

$126 \mid 2018$

Questions sur l'avenir du travail de mémoire

\title{
La banalité du mal à la croisée des regards. Philosophie, histoire, droit, cinéma
}

Vincent Lefebve

\section{(2) OpenEdition}

1 Journals

Édition électronique

URL : https://journals.openedition.org/temoigner/7327

DOI : $10.4000 /$ temoigner.7327

ISSN : 2506-6390

\section{Éditeur :}

Éditions du Centre d'études et de documentation Mémoire d'Auschwitz, Éditions Kimé

\section{Édition imprimée}

Date de publication : 2 avril 2018

Pagination : 104-121

ISBN : 978-2-930953-06-9

ISSN : 2031-4183

\section{Référence électronique}

Vincent Lefebve, "La banalité du mal à la croisée des regards. Philosophie, histoire, droit, cinéma ", Témoigner. Entre histoire et mémoire [En ligne], 126 | 2018, mis en ligne le 24 janvier 2022, consulté le 04 février 2022. URL : http://journals.openedition.org/temoigner/7327 ; DOl : https://doi.org/10.4000/ temoigner.7327 
La banalité du mal à la croisée des regards

Philosophie, histoire, droit, cinéma

a controverse engendrée par le livre de Hannah Arendt Eichmann à Jérusalem. Rapport sur la banalité du mal se terminera-t-elle un jour? Paru au début des années 1960', louvrage a immédiatement suscité une polémique internationale de grande ampleur (Cohen, 1993; Brudny et Winkler, 2001 Rabinbach, 2004). S'il a depuis fait régulièrement l'objet de commentaires, un regain d'intérêt notable vis-à-vis de ce livre s'est manifesté à partir du milieu des années 1990 et se poursuit aujourd'hui.

Cetécho contemporain à la controverse initiale suscitée par Eichmannà Jérusalem (ci-après « $E J »)$ présente diverses caractéristiques. En premier lieu, le recul temporel implique un changement dans le style et dans le ton : les discussions actuelles sont en règle générale moins passionnées, relèvent davantage du débat que de la polémique. En second lieu, alors que les réactions suscitées par louvrage dans les années 1960 se concentraient sur la notion de banalité du mal, ainsi que sur les observations d'Arendt se rapportant au rôle des dirigeants juifs dans le déroulement de la Shoah, on assiste depuis plusieurs années à un élargissement des thématiques abordées. En particulier, la pensée de la justice que recèle $E J$ retient l'attention des commentateurs, cette dernière s'inscrivant en réalité dans une philosophie du droit plus générale qui traversel'œuvre d'Arendt de part en part (Goldoni et McCorkindale, 2012 :Volk 2015; Lefebve, 2016). Enfin les positions d'Arendtsont appréhendées discutées a joul'hidepus desper EJrecoit une attention renouvelée de la part de philosophes, mais aussi d'historiens, de juristes, de psychologues et de cinéastes. Concernant cette dernière dimension, outre que le procès Eichmann, dès lors qu'il a été filmé et diffusé à la télévision, a constitué un événement médiatique singulier dont on n’a mesuré que récemment la portée (Lindeperget Wieviorka, 2016), il est particulièrement frappant que les thèses avancées par Arendt et plus généralement son intérêt pour le procès de Jérusalem aient fait l'objet d'adaptations à l'écran, soit que la position arendtienne inspire des réalisateurs de documentaires (Rony Brauman et Eyal Sivan, Un spécialiste. Portrait d'un criminel moderne, France, 1999), soit que le «personnage Arendt soit mis au centre d' Allemagne, 2012).

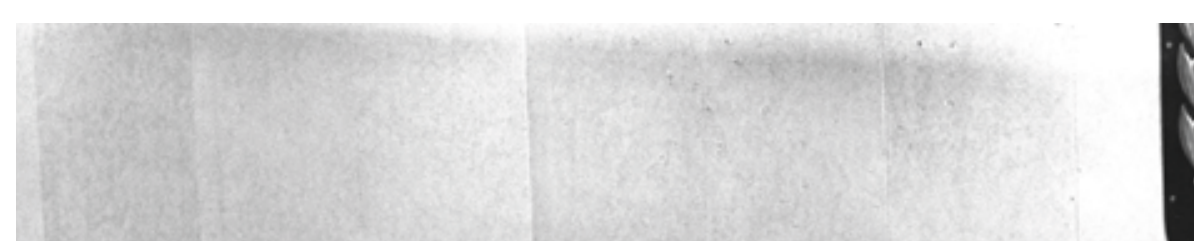

- Les trois juges qui ont Eichmann. De gaucheà à droite: Benjamin Halevi Moshe Landau et Yil
Raveh, 4 avril 1961
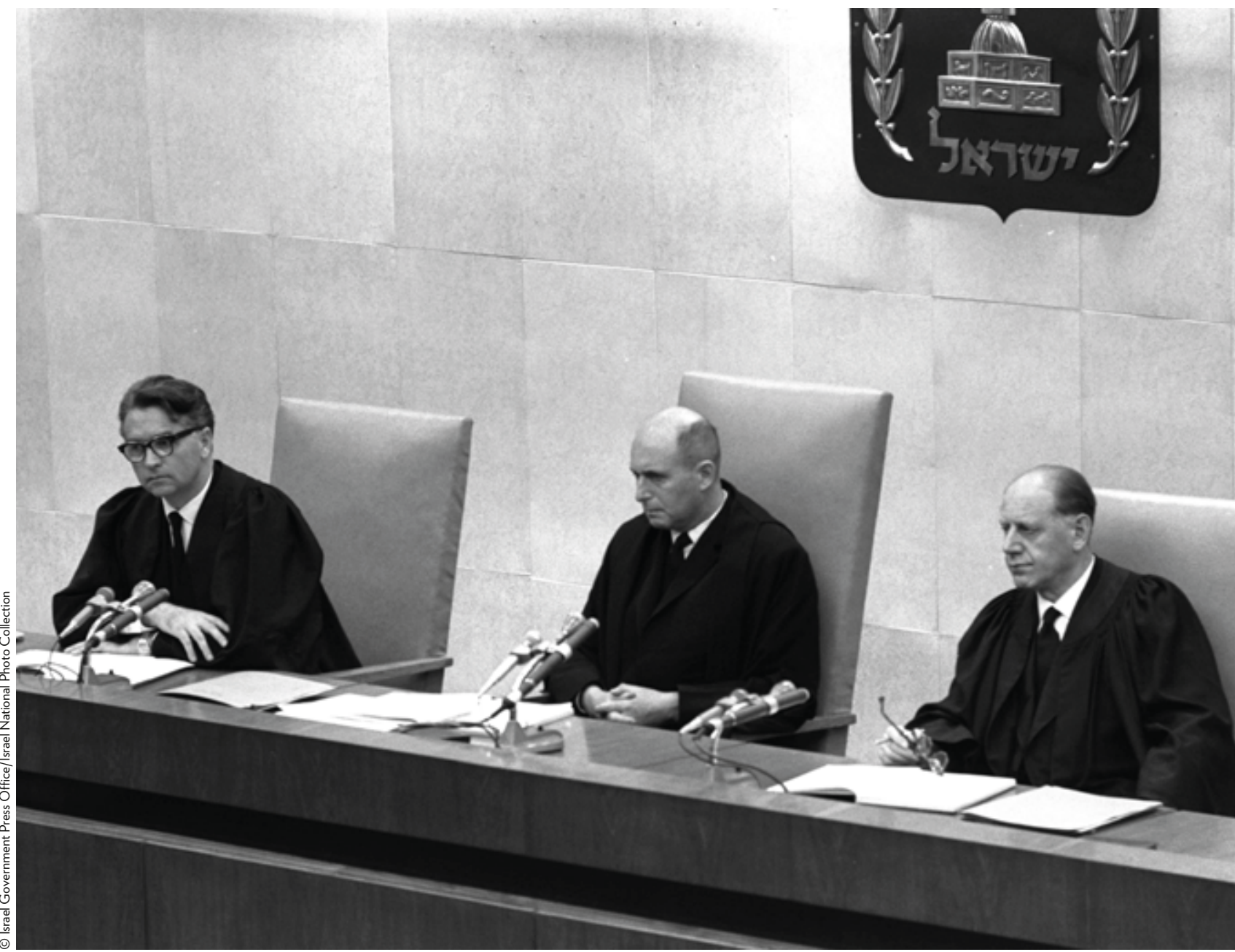
Si le potentiel heuristique de ce croisement de perspectives - philosophiques, historiographiques, cinématographiques, etc. - est indéniable, il est toutefois nécessaire que soit menée une patiente et délicate opération de réappropriation critique de ces diverses lectures d' $E J$. En effet, la pensée d'Arendt ayant fait l'objet depuis plus d'un demi-siècle d'interprétations ou de réappropriations contestables, qui n’en respectent ni la lettre ni l'esprit (Rousso, 2011, p. 20 ; Berkowitz, 2014), des précautions s'avèrent nécessaires. Un travail de contextualisation de ce livre dan l'œuvre générale de la philosophe doit en particulier être mené Ce n'est qu’à ce prix que ces lectures contemporaines peuvent révéler le potentiel de la pensée d'Arend pour affronter, dans leur specificite propre, certains phenomènes politiques contemporains, comme la montée en puissance dun terrorisme global qui est, par certain de ses aspects, comparable aux totalitarismes du XXe siècle (Lescourret et Godin 2016, p. 29-31; Garapon et Rosenfeld, 2016, p. 107).

\section{LA BANALITÉ DU MALA ÁÉPREUVE DE LHISTOIRE}

Parmi les travaux ayant réactivé la controverse ces dernières années, il convient de considérer en particulier les ouvrages de David Cesarani (2005) et de Bettina Stangneth (2016). Malgréla différence de leurs approches, ces auteurs partagentlacongneth (2016). Mréch tion que leurs recherches invalident les conclusions d'Arendt. Leurs ouvrages - par ailleurs remarquables - ont été écrits, en partie du moins, contre Arendt. Aux yeux de ces auteurs, et de la plupart de leurs commentateurs, la description d'Eichmann mise en avant par Arendt ne résiste pas à l'analyse de sources que la philosophe n’avait pas été en mesure de consulter à lépoque du procès ou qui avaient été mises partiellement à sa disposition.

Bettina Stangneth fait preuve d'une certaine bienveillance à l'égard d'Arendt. Rappelant le reproche qui lui est souvent formulé - n’avoir assisté « personnellement qu’à un faible nombre de journées du procès » (Stangneth, 2016, p. 571) elle reconnât qu'Arendt aété d'une des lectrices les plus méticuleuses des procèselle recon procèverbaux dinter en Amerique. (ibid.) Ne disposant pas de toutes les informations utiles, consciente du caractère parcellaire de sa compréhension de l'accusé, la philosophe a quand même voulu émettre un jugement, ce qui, d'une certaine façon, est tout à son honneur. Mais il faut bien admettre, estime Bettina Stangneth, qu'Arendt s'est fourvoyée : elle n'a pu comprendre qu' « Eichmann, à Jérusalem, était à peine plus qu’un masque. » (Stangneth, 2016, p. 20) Un constat similaire se retrouve sous la plume de David Cesarani, qui s'exprime souvent de façon plus critique encore que Bettina Stangneth.

Suite aux progrès engendrés par la recherche historique, $E J$ devrait-il être dès (2016, p. 492) En réalité, dans les deux cas, bien que suivant des modalités différentes, les préconceptions des positions arendtiennes qui semblent nourrir les travaux de Bettina Stangneth et de David Cesarani sont contestables. Si on lève les malentendus dans lesquels s'enferrent ces auteurs, on se rend compte que loin de mettre à mal les thèses d'Arendt, leurs recherches auraient plutôt pour effet de les renforcer.

Ces deux ouvrages indiquent en tout cas un déplacement dans les thématiques placées au cour de la controverse. Certains des points de focalisation de la polémique initiale, en particulier la question de la responsabilité des conseils juifs dans e déroulin une position centrale ${ }^{2}$. Cest la notion de banalité du mal qui est abordée, mais suivant un angle bien spécifique : la question de l'antisémitisme d'Eichmann est posée, et plus précisément encore celle de l'intensité de son antisémitisme.

Selon Cesarani, Arendt nierait, contre l'évidence, l'antisémitisme d'Eichmann : « De manière assez remarquable, étant donné toutes les preuves existantes du contraire, [Arendt] insista pour dire qu'Eichmann n’était pas antisémite et qu’il n’avait pas eu de motivation idéologique.» (Cesarani, 2010, p. 445)

Qu'en est-il dans $E J$ ? Arendt rappelle tout d'abord que personne n'a douté durant le proces du fait suivant : Eichmann « a toujours fait de son mieux pour rendre définitive la Solution finale.» (Arendt, 2002, p. 1039) Elle pose toutefois la question: était-ce là « une preuve de son fanatisme, de sa haine sans bornes à légard des Juifs? » (ibid.) Selon elle, « l'attitude sans compromis adoptée par Eichmann dans les dernières années de la guerre lui était probablement dictée non par son fanatisme mais par sa conscience même. » (ibid.) Eichmann n’a fait qu’appliquer a nouvelle loi du pays, c'est-à-dire la loi du meurtre, qui découlait de l'ordre du Führer, et il n’aurait eu mauvaise conscience que s'il ne s'y était pas soumis, « s'il n’avait pas exécuté les ordres - ordres d'expédier à la mort des millions d'hommes, de femmes et d'enfants, avec un grand zèle et le soin le plus méticuleux " (ibid)

Dans La Vie de l'esprit, Arendt précise encore sa pensée en indiquant qu'il n'y avait trace en Eichmann «ni de convictions idéologiques solides ni de motivations spécifiquement malignes.» (Arendt, 2005a, p. 21) On peut certes reconnaître qu'Arendt ne connaissait pas, dans toute son ampleur, l'intensité de l'antisémitisme d'Eichmann que révèlent les Papiers argentins ${ }^{3}$, une série de sources largement inédites que la philosophe et historienne allemande Bettina Stangneth a placée au centre de ses recherches (Berkowitz, 2014). Mais était-ce là véritablement l'objet de la question qui animait Arendt? Aurait-elle revu son jugement en prenant connaissance, dans tous leurs détails, des écrits et des enregistrements laissés par Eichmann en Argentine? Faut-il conclure aujourd'hui que les descriptions propośes dons $E J$ sont 2011, p. 169-170)
(2) En substance, Arendt reproche aux dirgeants 5 unfs
davoir pensé que coopererer avec les autorités du llle Reich rendrat de leur peuple. Elle estime que "s si le peuple juif avait vraiment été non organisé et dépourvu de direction, le chaos aurait régné, yaurait eu beaucoup de misere,
mais le nombre total des victimes naurait pas atteint quatre et
demia six millions, (Arendt. 2002, p. 1139) La position majoritaire dans la littérature est bien résumeé par Michelle-lresne
Brudny lorsyülle indique les « positions de Hannah Arend ne fécondent plus la recherche du rôle des conseils suifs dans la
date destruction des Juifs d'Europe ae été entièrement invalidée. "
(Brudny, 2016a, p. 47). Pour une approche plus nuancée de cette dimension d $d^{\prime} E J$, voir Leibovici et

(3) Parmi ces sources, il convien «entretiens Sassen », à savolir un série dentretiens entre Willem Sassen - un ancien membre Eichmann et des sympathisants conversations enregistrées bandes ainsi que leur retranscription écrite navaient
pas été exploitées de façon systématique. Certains extraits $d$ ces entretiens avaient toutefois été publiés dans le magazine
américain Life en 1960 . Arendt ayant pu en prendre connaissance au moment de rédiger $E$. 
-Adolf Eichmann, prison
d'Ayalon, ler avril $1961^{2}$

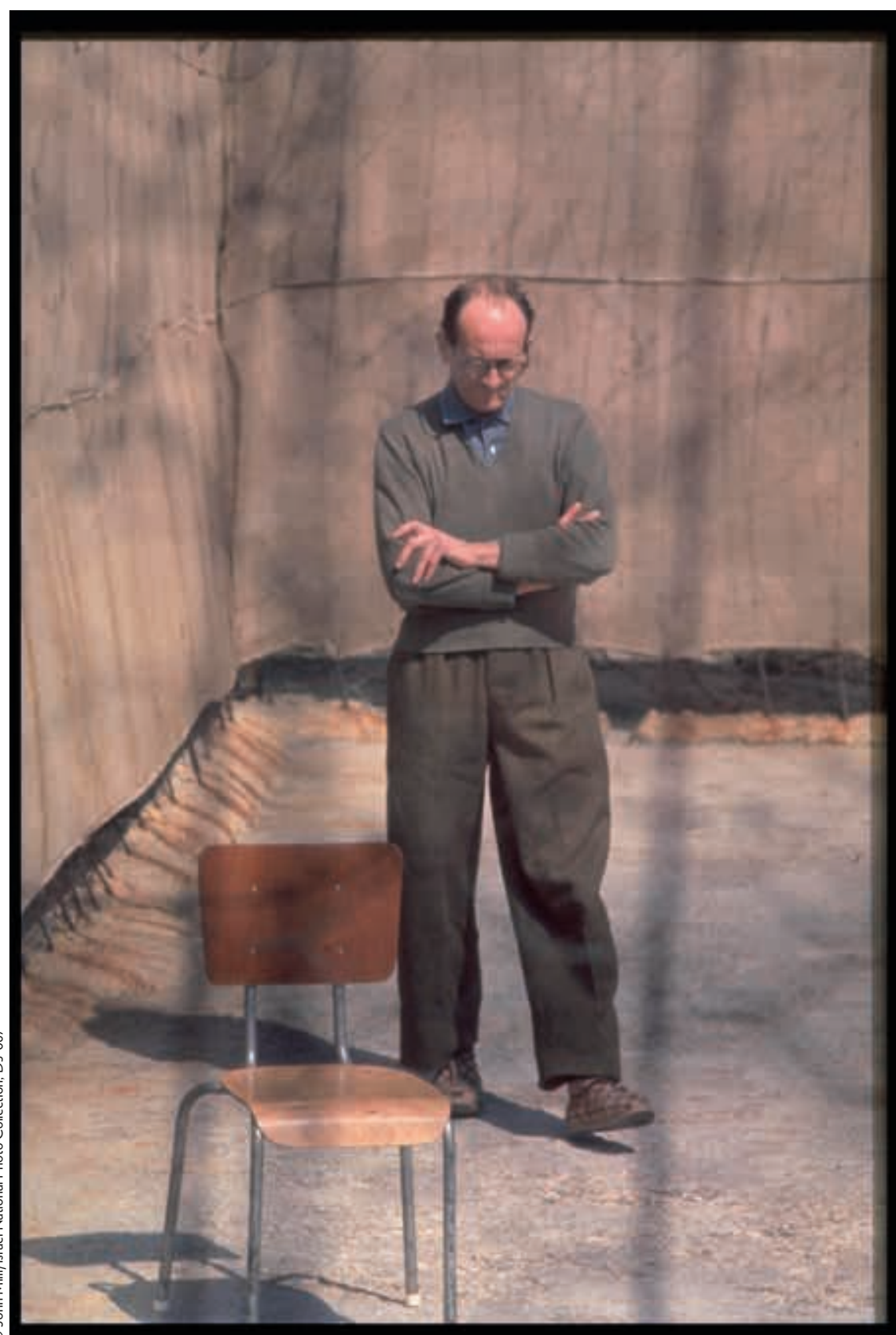

Arendt ne minimise pas l'antisémitisme d'Eichmann. Elle insiste plutôt sur le type d'antisémitisme qui est le sien, froid, objectif, se mettant au service du mouvement de la Nature (Arendt, 2004, p. 804 et 823). Dans cette variante idéologique, l'antisémitisme fait de l'existence des Juifs en tant que peuple un «problème » qui doit être réglé politiquement. Au lieu de la haine incandescente de celui qui commet le mal, Eichmann ressent ainsi la « joie "méta-sentimentale" d’avoir [...] participé en première ligne au Mouvement de l'Histoire mondiale. » (Leibovici et Roviello, 2017, p. 173) Plutôt que « des pulsions antisémites sadiques ou humiliantes ", il 2017, p. 173) pur affiche et me à l'édification d'un Nouveau Monde-le Reich 'mill métaphysiques.» (ibid., p. 173)

Bettina Stangneth confirme ces analyses. Comme elle le montre de façon détaillée dans son ouvrage, la « vision du monde » (Stangneth, 2016, p. 330) à laquelle adhère Eichmann est une « vision national-socialiste » (ibid., p. 163), qu’Eichmann a toujours défendue avec « virulence » (ibid.), qui postule un « combat final des races » ne pouvant cesser « tant qu’un adversaire est encore en vie. » (ibid., p. 331) En Argentine, Eichmann se sent orphelin de «la grande mission » (ibid., p. 198) qui était la sienne en Europe. L'antisémitisme auquel adhèrent l'ancien officier SS et était la sicter SS et ses inter. p. 423), vieux jeu, fondé sur la jalousie ou une animosite de type culturel, mais un " antisémitisme génocidaire totalitaire 》 (Leibovici et Roviello, 2017, p. 22). Ce dernier se fonde sur une « théorie raciale » fonctionnant en vase clos (Stangneth, 2016, p. 331) qui est en outre destinée à être appliquée, au-delà du peuple juif, au peuple allemand lui-même - à travers notamment le programme d'euthanasie dit Aktion T4 auquel Eichmann a, comme le note Bettina Stangneth, « apporté un soutien sans réserve. » (ibid., p. 333)

Le caractère objectif de cet antisémitisme se marque également dans le fait qu'Eichmann, s'il considère que «l'extermination des Juifs est une bonne chose » (ibid., p. 405), rapporte à plusieurs reprises qu'il « ne prend aucun plaisir à la confrontation directe avec l'angoisse, la torture et la mort des victimes. » (ibid.) Et s'il se définit non seulement comme un «bureaucrate prudent» (ibid.,p. 439), mais aussi comme un « combattant fanatique pour la liberté de [son] sang» (ibid.), ce n'est pas parce qu'il a pris un plaisir sensuel ou morbide aux tâches criminelles qui lui avaient été confiées, mais parce qu'il s'est engagé résolument dans l'accomplissement de sa mission : l'évacuation du peuple juif hors du territoire du Reich, et ensuite sa destruction physique. C'est là pour Eichmann tout à la fois un combat, une mission, un commandement, une loi qui revêtent à ses yeux une dimension sacrée (ibid. p. 427 et 439). 


\section{ABSENCE DE PENSÉE ET IDÉOLOGIE}

Bettina Stangneth serait-elle ainsi une arendtienne malgré elle ? Son livre « es aussi un dialogue avec Hannah Arendt » (ibid., p. 21), reconnait-elle, et elle rappelle que son «travail sur ce sujet a commencé [...] avec Eichmann à Jérusalem.» (ibid.) La philosophe et historienne allemande affirme toutefois s'être fondamentalement éloignée du point de vue arendtien, et ce en particulier s'agissant de la notion d'absence de pensée (thoughtlesness) à laquele se rattache conceptuellement la formule de (a) la banalité du mal. Selon Bettina Stangneth, les discours qu'Eichmann a pu teni avant et pendant son procès ne renvoient pas à un « bavardage irréfléchi » (ibid. p. 392), mais à « un discours coherent fondé sur un édifice intellectuel. » (ibid.) Bettina Stangneth identifie ainsi une forme de pensée chez Eichmann, et mêm une espèce de philosophie ${ }^{4}$

Tout se passe en réalité comme si les descriptions de Bettina Stangneth, loin d'invalider les thèses d'Arendt, avaient pour effet de les étayer. Car la forme de pensée qu’elle repère chez Eichmann n’est qu’un leurre, « un simulacre » (Leibovici et Roviello, 2017 p. 213). Eichmann retourne "contre la pensée un de ses moments fondamentaux après avoirévacuédecelle-cisa mesure interne:l'instance d'altérité jugeante. » (ibid.) Dans les nombreux écrits qu'il a signés avant et après sa capture en Argentine, Eichmann paraît en effet complètement dépourvu de la capacité de mener ce dialogue intime qu'Arendt assimile à la pensée, ou de se représente intérieurement, par la force de l'imagination, le mal qu'il a infligé à d'autres être humains ; la souffrance subie par autrui n'accède jamais, chez Eichmann, au statu d'« épreuve intérieure » (ibid., p. 214).

Il est ainsi particulièrement frappant que Bettina Stangneth déplore le caractère monologique de son discours :

En dépit de tous nos espoirs, se retrouver dans la solitude ne posait aucun problème à On aurait probablement pu enfermer cet homme pendant des décennics Eich san. aujourd hui celui qui lit ses textes. Il est facile et tentant de hausser les épaules face à ce bavardage inconsistant parce qui il constitue indubitablement, comme toute dogmatique, une mauvaise philosophie. Le fait est, cependant, que la logique de ces épouvantable échafaudages intellectuels était justement ce qui donnait à l'un des assassins de masse les plus efficaces de l'histoire l'assise mentale que nous aimerions tant voir mise à mal par le seul effet de la réflexion (Stangneth, 2016, p. 334).

Ces phrases de Bettina Stangneth entrent en résonance non seulement avec les descriptions de ce qu'est, selon Arendt, l'a qu'elle appelle dans Les Origines du totalitarismel'idélogie.

Dans une lettre à Mary McCarthy souvent citée (voir par exemple Cesarani, 2005, p. 445) Arendt énumère les éléments de son $E J$ qui, à son estime, « contredisent [...] le livre sur le totalitarisme.» (Arendt, 2002, p.1379) Parmi ceux-ci, elle affirme avoir «peut-être surestimé l'impact de l'idéologie sur l'individu.» (ibid.)

Ces remarques vont apparemment dans le sens de David Cesarani, BettinaStangneth et leurs commentateurs : ne disposant pas de suffisamment d'informations, Arendt n’aurait pas pris la mesure de l'idéologie à l'œuvre dans les actes d'Eichmann. Elle aurait été piégée par les apparences judiciaires édifiées par la défense d’Eichmann lors du procès, faisant de lui un homme ordinaire dépourvu de sentiments antisémites (Delpla, 2011, p.15).

Sil'on replace cette citation dans son contexte, on peut toutefois ouvrir une autre piste interprétative; Arendt ajoute en effet immédiatement

Même dans le livre sur le totalitarisme, je signale la curieuse perte de contenu idéologique qui affecte l'élite du mouvement. L'important, c'est le mouvement en lui-même; l'antisémitisme par exemple perd son contenu dans la politique d'extermination, car l'extermination n’aurait pas cessé s'il n'était plus resté un seul Juif à tuer. En d'autres termes, l'extermination per se est plus importante que l'antisémitisme ou le nazisme (Arendt, 2002, p. 1379)

Arendt se situe donc dans la ligne des descriptions de la notion d'idéologie qu'elle a proposées dans Les Origines du totalitarisme. L’idéologie est, comme le mot l'indique précisément, la «logique d’une idée» (Arendt, 2002,p. 825), idée à laquelle on attribue un pouvoir explicatif prodigieux quant au sens de l'histoire humaine, présente, passée et à venir, la capacité de résoudre tous les mystères de l'univers - cette prétention étant à mettre en lien avec le mépris profond de la mentalité totalitaire pour les faits et la réalité elle-même (ibid., p. 809).

Il faut donc prendre au sérieux Eichmann lorsqu'il indique avoir toujours obéi non seulement aux ordres, mais aussi à la loi, à condition de bien comprendre que le mot loi ne revêt pas ici son sens habituel, mais un sens national-socialiste. Dans les conditions du $\mathrm{II}^{\mathrm{e}}$ Reich, la loi suprême à laquelle Eichmann se soumet s'identifie à co volonté du Führer (ibid, p. 1150). Cette loi n’ pas pour dessein de stabiliser la vie la des êtres ho la fois eternel et inexorable de la Nature, cette dernière étant fondée sur une lutte à mort entre « races » antagoniques qualifiée par les dirigeants nazis de bataille destinale pour le peuple allemand (ibid., p. 1068).

Dès lors que le régime totalitaire doit être conçu, sur un mode dynamique, comme un mouvement dont il convient de percevoir en toutes circonstances la direction, il ne suffisait pas, sous le III ${ }^{e}$ Reich, d'exécuter scrupuleusement les ordres, ce qui renverrait à une vision statique de la relation entre gouvernants et gouvernés. Il était attendu, spécialement de la part des formations d'élite, davantage à savoir d'« exécuter la volontéde la direction (Arendt 2002 p. 733). Non obéir à la lettre (3) fait à laquelle ils s'appliquaient (ibid., p. 732-733; Kershaw 2000, p. 747-748). 


\section{LA THÉORIE DES ROUAGES}

On peut ici revenir sur la façon donc la perception d Eichmann par Arendt s'est fgée dans lesprit dun Cesarani. Si Arendt estime que la controverse initiale s'est développée à partir de l'image d'un livre et non d'un livre réel (Arendt, 2002, p. 1383 et 1412), les choses ne semblent pas avoir fondamentalement changé aujourd'hui. L'image d' $E J$ - qui s'est substituée au vrai $E J$ - est un ouvrage dans lequel serait postulée une théorie monolithique du totalitarisme. Dans le totalitarisme tel que pourat laurait thérise Arendt, et dont elle s'efforctit dans la personne die Théofilakis, 2013, p. 72), les ordres se diffuseraient du haut vers le bas, Eichmann pouvant dès lors être réduit à un «petit rouage » dans une machinerie bien huilée. Arendt est en réalité loin d'être la théoricienne d'un totalitarisme « monolithique ». Elle a très tôt pris au sérieux l'aspect déstructuré, chaotique de ce régime, spécialement dans sa version national-socialiste. Selon Arendt, la domination totalitaire renvoie à un régime qui se caractérise par la polycratie qu'il instaure et don il se nourrit (Arendt, 2002, p. 733-734). Elle écrit encore dans $E J$ : quiconque «a étudiéle totalitarisme [...] sait bien que le caractère monolithique de cette forme de gouvernement est un mythe. » (Arendt, 2002, p. 1166; Leibovici, 2011, p. 23)

Arendt était consciente que cette « théorie des rouages» (Arendt, 2005b, p. 70), qu’on lui attribue à tort, constituait un élément de la défense d'Eichmann : il était dans son intérêt d’être présenté comme un simple rouage dans une machinerie bien huilée, et ce afin que sa responsabilité soit amoindrie.

Tentant de déplacer la question, Arendt note qu'« il est dans la nature même du gouvernement totalitaire, et peut-être est-ce la nature de toute bureaucratie, de transformer les hommes en fonctionnaires, en simples rouages de la machine administrative et, ainsi, de les déshumaniser. On peut longtemps discuter et avec profit du règne de Personne qui est la vérité de la forme politique connue sous le nom de bureaucratie (Arendt, 2002, p. 1297) En d'autres termes, sile réme totalitaire est polycratique plutôt que monolithique, cela ne luifait pas perdre pou autant son caractère fondamentalement bureaucratique. Arendt loue d'ailleurs pour cette raison les travaux de Raul Hilberg, ce dernier ayant réussi dans La Destruction des Juifs d'Europe paru en 1961, «à établir la première description claire de [la] machine de destruction [nazie], dans toute son incroyable complexité. » (Arendt, 2002, p. 1088)

Cependant, si Arendt reconnait que la « théorie des rouages » peut expliquer, dans une certaine mesure, le fonctionnement d'un système, qu'il soit totalitaire ou plus simplement bureaucratique, elle affirme que le grand avantage de la procédure judiciaire qui explique en outre la «grandeur» du mécanisme même des poursuite judiciares (Arendt, 2005b, p. 201), est qu'elle nacorde acune inpotanc ges. L’accusé placé au centre d'un procès est considéré comme un individu responsable de ses agissements et tenu de rendre des comptes; dans l'enceinte d'un tribunal, le rouage est à nouveau appréhendé comme une personne (Lefebve, 2016, p. 213).

Cette insistance proprement arendtienne sur la personnalisation des comportements inhérente à l'œuvre de justice renvoie à une autre dimension de sa philosophie du droit: sa conception moniste, plutôt que pluraliste, des buts assignés à un procès. Selon Arendt, le but d'un procès, aussi exceptionnel soit-il, n'est en effet pas de servir la raison d'État ou d'écrire l'histoire, mais de dire le droit et de "rendre justice" (Arendt, 2002, p. 1263) La tâce quice » (Arendt, 2002, p. 1263). La tâche qui incombait au tribunal de Jêrusalem était donc la suivante peser les arguments de 'accusation : per la defs par les parties, mener les débats et, finalement, rendre un verdict. Un verdict qui concerne une personne singulière, non un système politique, une idée abstraite ou une tendance historique (Arendt, 2005b, p. 57-91 et p. 71-72).

Le recul historique peut nous inciter à nuancer ces affirmations d'Arendt (Leibovici et Roviello, 2017, p. 78). Il s'avère en effet aujourd'hui difficile de nier les effets politiques des procès pour crimes de masse et, en particulier, «leur pouvoir public de révélation et de mémoire " (ibid ; voir aussi Osiel, 2006 et Felman, 2001). Avec Claude Claude Klein, on peut postulerquin ; plusieurs objectifs, Iun de facture classique, visant à établin) accusé, lautre de portée plus historique (Klein, 2012, p. 135).

Cependant, dans un registre plus positif, la position arendtienne a le mérite de ne pas faire de la justice un simple instrument au service de buts politiques extérieurs et prétendument plus élevés. S’agissant de la justice pénale internationale, en particulier, le fait que la philosophe insiste tant sur le thème de l'autonomie du processus de justice permet de la distinguer des auteurs contemporains qui voient dans la justice pénale internationale avant tout un instrument mis au service d'autres visées (la réconciliation, la pacification, l'éducation des populations impliquées dans un conflit, etc) Une telle conception a lon outre le mérite de nous interpeller aujourd'hui, alors etc.). Une tello cons qu'une place de plus en plus importante, voire dominante, est conferee aux victimes dans le cadre des procédures pénales, internationales ou nationales (pour une dis-
cussion de ces questions, voir Luban, 2011; Lefebve, 2015 ; Delpla, 2016).

\section{REGARDS CINÉMATOGRAPHIQUES SUR LA BANALITÉ DU MAL}

Dans cette section conclusive, je propose d'analyser trois œuvres cinématographiques afin de mettre en lumière differentes modalités dans la réception et la perception de l'œuvre d'Arendt, notamment auprès du grand public. Tout d'abord, j'illustre grâce au film Eichmann de Robert Young une conception traditionnelle du mal. Suivant cette dernière, le mal prend ses racines dans une intentionnalite Ju partie $E J(a)$ 
J'aborde ensuite, à partir du documentaire Un spécialiste de Rony Brauman et Eyal Sivan, un exemple de réappropriation problématique de la pensée arendtienne (et, par la même occasion, du procès Eichmann), en l'occurrence non par des détracteurs, mais par de prétendus continuateurs de la philosophe (b).

Avec le film Hannah Arendt de Margarethe von Trotta, j'examine une tentative plus aboutie, bien que souffrant elle aussi de certaines imperfections, de mettre en scène et en lumière la pensée d'Arendt à travers le septième art (c).

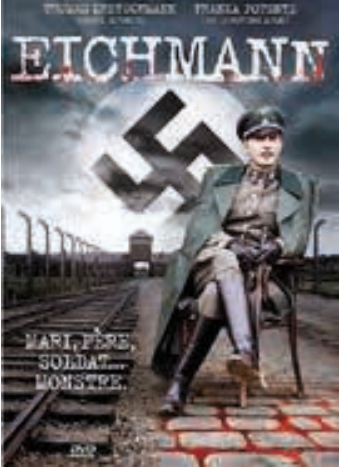

a Eichmann de Robert Young: la conception traditionnelle du mal

En écrivant $E J$, Arendt s'est efforcée « de détruire la légende de la grandeur du $\mathrm{mal}$, de sa force démoniaque, de retirer aux gens l'admiration qu'ils ont pour de grands malfaiteurs comme Richard III. » (Arendt, 2007, p. 149) Même si le nom d'Arendt n'est jamais mentionné, on trouve dans le film de Robert Young consacré à Adolf Eichmann une représentation particulièrement évocatrice de cette conception traditionnelle. L'intrigue du film se centre sur l'interrogatoire d'Eichmann qui s'est tenu en Israël, avant le procès, mené par le capitaine de police Avner Less. Les dialogues du film, est-il affirmé au début de celui-ci, s'inspirent du compte-rendu de cet interrogtoire. Le titre bref du film Eichmann est compléte sur lo fich

Le scénario vise à explorer un personnage fondamentalement double : « d’un côté, la bête sadique et ses crimes avant 1945 ; de l'autre lêtre humain et les côtés sympathiques qu'il manifesta lors de la captivité. » (Steinle, 2016, p. 270) Concernant la première facette de cet Eichmann au double visage, et à la suite de Matthias Steinle, deux scènes méritent en particulier d'être rapportées. Dans la première, une baronne imaginaire, de nationalité hongroise, nous est présentée comme la maitresse d'Eichmann. Se trouvant dans son château, en haut d'un majestueux escalier, elle se dévêtit et s'approche de son amant Se comportant de facon langoureuse, elle demande alors à Eichmann, en uniforme SS, combien de Juifs il a tués. Suit une (n) Suit une durexacte sur le plan historique, durant laquelle lèvocation de la Shoah est censée provoque une augmentation du désir des deux amants enlacés. Dans la seconde scène, qui suit immédiatement cette séquence, un bébé atteint, nous est-il dit, d’une «maladie héréditaire du sang » est arraché à sa mère, placé dans un panier, et emporté par la baronne; on entend longuement sangloter la mère de l'enfant. La baronne se rend auprès d'Eichmann, toujours en uniforme, et elle lui demande alors de rendre au Reich le « service » qu'impose une telle situation. Eichmann s'exécute et assassine froidement le nouveau-nea al'aide d'un pistolet, sous le regard horrifié de sa secrétaire.

On peut suivre ici le commentaire de Matthias Steinle : «Au-delà d'une provocation bon marché et malsaine, ces représentations permettent de construire une dramaturgie de la destruction des Juifs et de creuser l'écart entre le "soldat, monstre" et le "mari, père" évoqué dans le sous-titre » (ibid., p. 272) Elles nous ramènentégalement, père évàué értes caricaturale à la conception traditionnelle du mal évoquée plus haut, selon laquelle ce dernier a inévitablement partie liée avec des motivations intimes d'une certaine complexité, éventuellement sexuellement connotées, sordides ou diaboliques. Or, l'un des problèmes posés par le cas d'Eichmann, qu'Arendt avait d'ailleurs clairement identifié, était que l'ancien officier SS et grand organisateur de la Shoah, qui n’a d'ailleurs jamais tué personne de ses propres minel était-il donc?

Un spécialiste. Portrait d'un criminel moderne de Rony Brauman et Eyal Sivan : la banalisation de la banalité du ma

C'est à cette question qu'entend répondre le documentaire Un spécialiste de Rony Brauman et Eyal Sivan, ces derniers ayant ouvertement revendique l'heritage d'Arendt. Avant d'en venir à une analyse de ce film, on peut tenter de revenir sur le « contresens complet » (Brudny, 2016b, p. 224) qui s'est opéré dans l'interprétation et la réception de la formule arendtienne de la banalité du mal. Le déplacement qui està l'œuvre est celui de la banalitédu mal dont parle Arendt, en direction de l'idé suivant laquelle la banalité affecterait celui qui commetle mal, voire que la philosophe suivant laquelle la banalite affecterait celui qui commetle mal, voire que
aurait encouragé une «banalisation des crimes eux-mêmes » (ibid. $)^{5}$.

Telle est exactement la perspective qưon retrouve dans Un spécialiste qui, vouTelle est exactement la perspective qưon retrouve dans Un spécialiste qui, vou-
ant explorer la thématique de la banalité du mal à partir des images d'archive du procès, finit par réduire Eichmann à un « personnage banal » (Brauman et Sivan, 2006, p. 12). Dans l'ouvrage qui accompagne le film, et même sur l'affiche de celui-ci, les auteurs revendiquent une filiation à l'égard de la pensée d'Arendt (ibid., p. 9). Il s'agit là d'une des raisons qui justifient, selon les auteurs, le caractère libre de leur travail de montage, d'utilisation et de réappropriation des archives du procès, qui équivaut pour certainsa anne pure et simple falsification (Truster, 2016; Niney, 2016).

Dans Un spécialiste, tout est mis en place pour nous montrer qu’Eichmann est

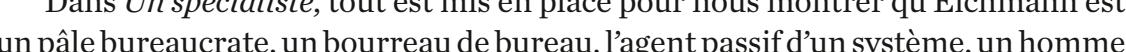
un pâle bureaucrate, un bourreau de bureau, l'agent passif d'un système, un homme
ordinaire. Il s'agit de bien saisir que cet « homme qui nous raconte son travail, nous ordinaire. Il sagit de bien saisir que cet « homme qui nous raconte son travail, nous
parle de ses joies et de ses peines, ressemble [...] a tout le monde.» (Brauman et Sivan, parle de ses joies et de ses peines, ressemble [.... à tout le monde.»(Brauman et Sivan, 2006, p. 98) L'un des objectifs du montage est de susciter, dans le chef du spectateur, remarques critiques de Lacoste, 2010, p. 399-400). De là, il n'y a qu'un pas à franchir pour affirmer qu’un petit Eichmann, « un génocidaire potentiel sommeillerait dans les bureaucrates et managers zélés, voire en chacun d'entre nous. » (Delpla, 2011, p. 14) Une telle conclusion, à zaquelle Arendts'stelle-même explicitementoppose (Arendt 2002, p. 1412-1414; Arendt, 2007, p. 92), constitue désormais un élément a part entière de la « véritable lieu commun contresensique et désémantisé » (Brudny, 2016, p. 224).

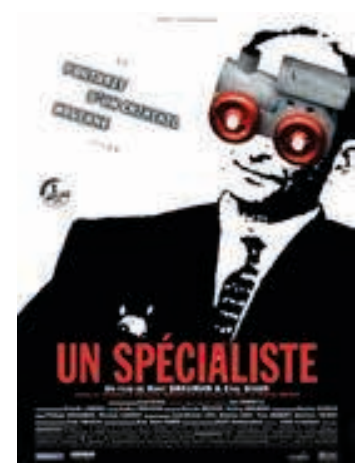
(5) Outre le fait quiArendt
a qualifié la Shoah de uplus grand malheur w du XX's siècle
(Arendt, 2002, p. 1412) eta vu en Eichmann « un des plus
grands criminels de son époque , grands criminels de son époque de forger des outils théoriques pour penser le caractère sans precedent du totalitarisme et
des crimes contis Elle sáttache ainsi, dans $E J$, à proposer une définition originale du génocide comme crime contre
lhumanite, au sens d'une atteinte portée à la pluralité humaine, " une attaque contre la diversite à-dire contre une caractéristique du "statut dêtre humain" sans lequel les mots mêmes de
"genre humain" ou d"humanite nauraient plus aucun sens. " (ibid,., p. 1277) Voir Butter, 2013, 
L’analyse d'Un spécialiste peut ainsi conduire à poser une question de portée plus générale, qui apparaissait déjà en filigrane des considérations qui précèdent pourquoi la pensée d'Arendt, exprimée dans $E J$, fait-elle l'objet de tant de malentendus? Outre les authentiques lectures paresseuses ou malveillantes auxquelles se livrent certains auteurs, il faut reconnaitre que l'ouvrage d'Arendt est sans doute lui-même à l’origine de certaines difficultés. $E J$ « n'est pas servi par la rapidité voire les raccourcis de certains passages.» (Leibovici et Roviello, 2017,p.9) Arendt préfère souvent aux formules directes et sans ambages le a second degré, comme si elle présupposait une complicité implicite avec le lecteur.» (ibid.)

Toutefois, les raisons profondes de cette réception problématique ne sont san doute pas internes à l'œuvre d'Arendt. Comme cela a été récemment mis en lumière par Martine Leibovici et Anne-Marie Roviello, une partie de l'explication peut être recherchée dans l'identification qui a rapidementétéopérée entre les thèses d'Arend et celles de Stanley Milgram (Milgram, 1994). La célèbre expérience mise au poin par le psychologue américain, au début des années 1960, tout en prétendant s’inscrire dans la continuité des découvertes d'Arendt, a en effet « joué un rôle important dans la réception déformée des analyses arendtiennes.» (Leibovici et Roviello, 2017. p. 33) culture populaire, par exemple dans le film I... comme Icare d'Henri Verneuil (France, 1979). Pour rappel, dans le cadre de cette expérience - ou plus exactement dans le cadre de sa variante principale -, des individus (en réalité des cobayes) sont invités à participer à une expérience scientifique (en réalité simulée) visant à tester la mémoire de sujets. Ces derniers doivent retenir des listes de mots et sont soumis, en cas d'erreur à des décharges électriques, de plus en plus fortes, que sont

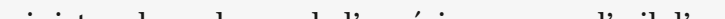
laboratinen bloseblanche (uncomplice de Milgram) Cereptsentant du monde scientifure a reçu deux consignes principales : d'une

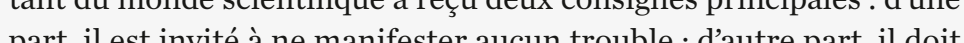
part, il est invitè à ne manifester auclun trich ; dautre part, il doit rappeler aux cobayes la nécessité de mener l'expérience à son terme en en respectant le protocole. Le résultat de l'expérience, dans sa variante principale à tout le moins, est que les cobayes, dans leur grande majorité, et même s'il leur arrive d'exprimer des réticences, accomplissent les actes qu’ils se sont engagés à poser. Ils torturent ainsi des innocents au nom de prétendus objectifs scientifiques.
Les conclusions de Milgram peuvent être résumées comme suit : à condition qu'une figure investie de l'autorité leur enjoigne, les êtres humains sont capables de n'importe quelle action. L'hypothèse sous-jacente est celle d'une propension naturelle de lêtre humain à l'obéissance. Selon certains auteurs (Goldhagen, 1996, p. 23 ; Cesarani, 2005, p. 451; Delpla, 2011, p. 13-15, 127-129 et 135), mais aussi pour les réalisateurs d'Un spécialiste (Brauman et Sivan, p. 14 sq.), cette thèse serait également celle d'Arendt : cette dernière aurait offert, dans $E J$, le portrait d'un Eichmann qui, à l'image d'un robot sans âme, exécuterait machinalement les ordres donnés sans se les réapproprier intimement.

L'écart entre les conceptions d'Arendt et de Milgram est pourtant considérable tandis que l'une souhaite mettre en lumière les conditions qui permettent l'émergence de la liberté et de la responsabilité humaines, Milgram et ses émules prennent au piège la liberté « dans leur lit de Procuste déterministe » (Leibovici et Roviello, 2017, p. 43), la considérant comme un simple « vernis » qui masque une nature inquiétante, celle de la soumission à l'autorité (ibid.).

c Hannah Arendt de Margarethe von Trotta : prendre la pensée au sérieux

La traduction en anglais du livre de Bettina Stangneth, en 2014, a donné lieu à une renaissance de la controverse aux États-Unis. Une querelle philosophique à une renaisso de s'est notanme élevee entre Seyla Benhabibet Richard Wolin. L'une défend la thèse de la prépondérance de Phéritage kantien dans linterpretation arendtienne de la notion d'« absence de pensée », en faisant fond sur l'intérêt tardif d'Arend pour la troisième critique kantienne, la Critique de la faculté de juger (Benhabib, 2017 ; Arendt, 1991). Richard Wolin défend une autre thèse, celle d'une reprise, à travers la notion de thoughtlessness, de la philosophie de Heidegger. Richard Wolin reproche également à Seyla Benhabib de perpétuer le mythe d’une Arendt dotée d’une perspicacité souveraine - quand tant de ses contemporains ségarent-, ardente défenseuse de la vérité - quand tant d'autres versent dans l'erreur-, seule contre tous. Il estime qu'une telle représentation apologétique est également celle que mave met en ava Michele-Irene Brudny partage cette conviction. Elle écit quelefilt de Margarethe von Trotta propose une «version hagiographique » de la polémique initiale (Brudny, 2016a, p. 47) et se risque à poser cette question, qu'elle laisse toutefois en suspens : pourquoi une telle présentation a-t-elle surgi à un tel moment de l'histoire et à un tel moment de la réception d'EJ? (Brudny, 2016b, p. 224)

Dès lors que la pensée d'Arendt faitl'objet de travestissements voire d'un renversement complet, qu'on la fige dans un slogan («si le mal est banal, c'est parce qu’i peut être commis par chacun d'entre nous, tout dépend des circonstances u) qui pent editl'essence de philo hie n'est-il pas compréhensible que des excices de réhabilitation puissentêtre de rehabilitation 
ainsi que la plupart des contributions que l'on retrouve dans Golsan et Misemer, 2017). Dans un autre registre, esthétique, le film de Margarethe von Trotta a éte explicitement conçu et présenté de cette façon, comme une défense d'Arendt.

Si certains de ses passages s'avèrent problématiques, en conduisant à une certaine simplification voire déformation du propos d'Arendt - malgré une utilisation abondante, et parfois un peu pesante, des sources arendtiennes (conférences, correspondance, etc) comme texture des dialogues - le film atteint, de façon intéresrespontessante, certains des objectifs quili s'est fixés. En particulier, Margarethe von Trotta, au moyen de divers procédés cinénatographiques, parvientà nontrer ou à tout le moins suggérer ce quest une pensée en acte. et invisibilité (Hyvönen et Möller, 2017)

Ayant décidé de se centrer sur l'événement qu’a constitué le procès Eichmann, elle révele en outre l'un des traits centraux de la méthode arendtienne: penser non seulement librement, «sans garde-fou » (Arendt, 2007, p. 128), mais aussi penser à partir d'événements, en s'y nourrissant, en y revenant sans cesse. En usant de l'un de ces aphorismes métaphoriques qu'elle affectionnait tant, Arendt a un jour parfaitement résumé cette caractéristique essentielle de sa méthode : La courbe que décritl cé é décrit lactivité de pensée doit rester liée à lévénement comme le cercle reste lié son foyer. » (Arendt citée par Habib, 1989, p. 7) On pent dise du film de Margarethe von Trotta qu'il parvient à décrire attachée sa vie durant à dessiner.

La cinéaste s'emploie en outre à rendre vivante une controverse philosophique et a mettre en evidence la façon dont elle se mêle à une campagne politique. Rappele qu'Arendt a fait l'objet d'attaques virulentes, parfois ignominieuses, ne revient pas à lui conférer une dimension mythologique. À l'époque de la controverse initiale, certains n'onten effet pas hésité àceler dans son livre une tentativededisculpation d'Eichmann, voire une affirmation de ses propres convictions nazies.

Plus fondamentalement encore, le film de Margarethe von Trotta parvient à souligner une dimension importante de la philosophie d'Arendt, celle de la responsabilité humaine de juger. Loin de considérer que nous serions tous des «Eichmann en puissance» (Chapoutot, 2017, p. 217), Arendt propose en effet de refonder la morale à partir du constat to 2017, p. 217), Arendt propose en effet de refonder la nus que la situation des Justes, elle s'intéresse aux «non-participants », à ces personnes qui «n'ont pas collaboré et ont refusé de participer à la vie publique, bien qu’elles n’aient pu se révolter et ne l'aient pas fait.» (Arendt, 2005b, p. 86) Ces individus, qui ont osé juger par eux-mêmes, n’y sont pas parvenus parce que leur système de valeurs étai meilleur ou plus solide que celui de ceux qui les entouraient, mais parce qus etait sont demandé dàn ces personnes pensaient, au sens qu'Arendt attribue à ce mot, celui d'un dialogue silencieux que l'esprit mène avec lui-même (Arendt, 2005a).

Suivant des modalités qui ne pourraient être explicitées ici (voir Lefebve, 2016, p.184-210), la faculté de pensée entretient des liens intimes avec une autre faculté, le jugement. Ce dernier « réalise la pensée, la rend manifeste dans le monde des phénomènes où l'on n'est jamais seul et toujours trop occupé pour penser. La manifestation du vent de lapensé n'est pas le savoir; c'estl'ptitude à distinguer le bien du matl le ver le bien dumal, lebendulidic mann semblait aux yeux dArendt déponivu de jugen une situation en embrassant le point de vue dautrui (Arend, 2002, p. 1064), ce qui explique sa tendance à se réfugier derrière le langage bureaucratique et à user de clichés (ibid., p. 1064 et 1065). Eichmann se cramponnait à la décision résolue qu’il avait prise de ne pas juger, de ne pas porter d'évaluation sur «le contenu même de ce qu' [indiquait] la volonté du Führer. » (Leibovici et Roviello, 2017, p. 267) Aussi, lorsqu'Arendt affirme ne pas percevoir chez Eichmann de convictions idéologiques fermes, elle veut indiquer qu'il n’était mû «par aucune conviction propre», toujours prêt « à troquer une conviction contre une autre au gré des changements de la ligne du Parti ou du sens attribué au Mouvement. (ibid., p. 268)

En dépit de ses maladresses, le film de Margarethe von Trotta constitue indubitablement un éloge du jugement. En cela, il prolonge la pensée d'Arendt et invente de nouvelles modalités de réinscription de celle-ci dans notre monde contemporain. La banalité du mal n’a pas encore dit son dernier mot. 
BIBLIOGRAPHIE

- Hannah Arendt, Juger. Sur la philosophie politique de Kant, trad. Myriam Revault d'Allonnes, suivi de deux essais interprétatiffs par Ronald Beit

- Les Origines du totalitarisme. Eichmann à Jérusalem, [1951 et 1963]. Pierre Bouretz (dir).), traductions entièrement révisées (Les Origines
du totalitarisme Mu totalitarisme, trad. Micheline Pounead [Sur IAntisémitisme], et Patrick Lévy [Le système totalitaire], révision Hélène Frappat ; Eichmann à Jérusalem, trad. Anne Guérin et Michelle-lrène Brudnyde Launay, révision Martine Lelbovici), Paris, Gallimard, " Quarto ",
2002.

- (a) La Vie de Pesprit [1978], trad. Lucienne Lotringer, Paris, PUF,
"Quadrige, ,2005.

- (b) Responsabilití et juggement, [2003], trad. Jean-Luc Fidel, Paris,
Payot \& Rivages, « Petite Bibliothęque Payot », 2005. - Edififir un monde. IIterventions 1971-1975, trad. Mira Köller et
Domininue Séglard, Peris, Seuili, « Traces écrites », 2007.

- Seyla Benhabib, «Whose Trial? Adolf Eichman's or Hannah Arendt's? The Eichmannn, Controversy Revisited », in Richard J. Golsan et Sarah
M. Misemer (ed.), The Trial That Never Ends: Hannah Arendt's Eichma in Jerusalem in Retrospect, Toron
Toronto Press, 2017, p. 209-228.

- Roger Berkowitz, The Good Society, vol. 23, n’ 2, 2014, p. 193-205. - Rony Brauman et Eyal Sivan, Éloge de la désobéissance. À propos d'« un

- Michelle--lìne Brudny et Jean-Marie Winkler (dir), Destins de «l la - Michelle-lrène Brudny (2016a), « Eichmann à Jérusalem ou la

- Michelle-lrène Brudny (2016b), «Eichmann à Jérusalem : confection, (dir), Le Moment Eichmann, Paris, Albin Michel, p. 203-226.

- José Brunner «E Eichmann's mind: Psychological, philosophical and legal
perspectives «, Theoretical Inquiries in Law, vol. 1, 2000, p. 429-464. - Judith Butler, Vers la cohabitation. Judéité et critique du sionisme, Paris,

- David Cesarani, Adolf Eichmann, trad. Olivier Ruchet, Paris, Tallandier,
2010 .

Johann Chapoutott, La Révolution culturelle nazie, Paris, Gallimard,
"Bibliothèque des histoires ", 2017. - Richard I. Cohen, «Breaking the Code: Hannah Arendt's Eichmann
in Jerusalem and the Public Polemice Myth Memory and Historical in Jerusalem and the Public Polemic: Myth, Memory and Historical

- Isabelle Delpla, Le Mal en procès. Eichmann et les théodicées modernes,
Paris, Hermann, « Lavocat du diable „, 2011.

- Isabelle Delpla, « Pythie ou témoin? ? Arendt, une vision datáe de la
justice internationale \#, Cités, vol. $67, n^{\circ}$ 3, 2016, p. $105-116$. - Emmanuel Faye, Arendt et Heidegger. Extermination nazie et destructio
de la pensée, Paris, Albin Michel, «ldées », 2016.
Shoshana Felman, «Thêatres de justice: Hannah Arendt à Jérusalem.
Le procés Eichmann et la redéfinition du sens de la loi dans le sillage de Le procès Eichmann et la redéfinition nu sens dela loo dans le sillag
l'holocauste ", Les Temps modernes, vol. 515/516, 2001, p. 23-74. Antoine Garapon, et Michel Rosenfeld, Démocraties sous stress. Les défis du terrorisme global, Paris, PUF, 2016.

Daniel J. Goldhagen, Les Bourreaux volontaires de Hitter : les Allemands ordinaires et ' 'Holocauste, trad. Pierre Martin, Paris, Seuil « « Points

- Marco Goldoni et Christopher McCorkindale (ed.), Hannah Arendt and
the Law, Oxford, Hart Publishing, «Law and Practical Reason », 2012. - Richard J. Golsan et Sarah M. Misemer (ed.), The Trial That Never Ends. Hannah Arendt's Eichmann in Jerusalem in Retrospect, Toronto/Buffalo/

- Claude Habib (dir), , Introduction », in Hannah Arendt, Penser
l'événement, Paris, Belin, 1989, p. 7-12.

- Annabel Herzog (coord.), Hannah Arendt. Totalitarisme et banalité du
mal, Paris, PUF, « Débats philosophiques », 2011. Raul Hilberg, La Destruction des Juifs d'Europe [1961], trad. André

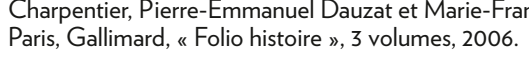
- Ari-Elmeri Hyvönen et Frank Möller, «V Visualising political thinking on the screen: a dialogue between von Trottás Hannah Arendt and its
protagonist, J Journal for Cultural Research, vol. 21, $\mathrm{n}^{\circ} 2,2017, \mathrm{p}$. 140protage
156.

- lan Kershaw, Hitter. Tome 2. 1936-1945, trad. Pierre-Emmanuel Dauzat,

- Claude Klein, Le Cas Eichmann. Vu de Jérusalem, Paris, Gallimard, 2012. - Charlotte Lacoste, Séductions du bourreau, Paris, PUF, « Intervention
philosophique », 2010.

- Vincent Lefebve, « Hannah Arendt et le problème de la justice pénale internationale. Une pensée toujours actuell
$d ' E$ Études suridiques, vol. 75, 2015, p. 27-58.

- Vincent Lefebve, Politique des limites, limites de la polititiue. La place du

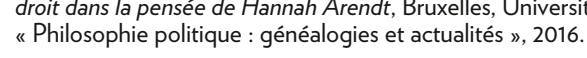

- Martine Leibovici, « De lobeíssance en régime totalitaire », in Annabel
Herzog (dir.), Hannah Arendt. Totalitarisme et banalité du mal, Paris, PUF, "Débats philosophiques », 2011, p. 15 à 36.

- Martine Leibovici et Anne-Marie Roviello, Le Pervertissement totalitaire.
La banalité du mal selon Hannah Arendt, Paris, Kimé, 2017.

.

- Marie-Anne Lescourret et Christian Godin, «La condition de
linhumain », Cités, vol. 67, n⿳亠口冋. 3 , 2016, p. 19-36.

- Sylyie Lindeperg et Annette Wieviorka (dir.), Le Moment Eichmann
Paris, Albin Michel, 2016 .

Deborah E. Lipstadt, The Eichmann Trial, New York, Nextbook
Schocken, 2011.

- David Luban, « Hannah Arendt as a Theorist of International Crimina
Law w, International Criminal Law Review, vol. 11, 2011, p. 621-641.
FILMOGRAPHIE

- Stanley Milgram, Soumission à 'autorité [1974], trad. Emy Molinié, Paris, - François Niney, « Reprise de vues, rigueur historique et licence poétique : à propos dun remake du procès Eichmann ", in Sylvie
Lindeperg et Annette Wieviorka (dir.), Le Moment Eichmann, Paris

- Mark Osiel, Juger les crimes de masse. La mémoire collective et le droit
[1997], trad. J.-L. Fidel, préf. Antoine Garapon, Paris, Seuil, «L La couleur

- Anson Rabinbach, «Eichmann in New York: The New York Intellectuals - Henry Rousso, Catalogue ex Juger Eichmann, Jérusalem, 1961 1, Paris, Mémorial de la S1
issaéliennes, 2011.

- Bettina Stangneth, Eichmann avant Jérusalem. La vie tranquille d’un
génocidarie, trad. Olivier Mannoni, Paris, Calmann-Lévy, 2016.

- Matthias Steinle, « Adolf Eichmann sur les écrans allemands: entre
docu-drama et docu-fiction, in Sylvie Lindeperg et Annette Wieviork docu-drama et docu-fiction, in Sylvie Lindeperg et Annette Wieviorta
(diri.), Le Moment Eichmann, Paris, Albin Michel, 2016, p. 259-281. - Fabien Théofilakis, «Adalf Eichmann à Jérusalem ou le procès vu de la
cage de verre (1961-1962) », Vingtième Siecle. Revue d'histoire, vol. 120 ,
2013, p. 71-85.

- Stewart Tryster, « De Pusage de 'archive dans Un spécialiste », in Sylvie
Lindeperg et Annette Wieviorka (dir.). Le Moment Eichmann, Paris. Albin Michel, 2016, p. 227-245.

- Christian Volk, Arendtian Constitutitionalism. Law, Politics and the Order
of Freedom, Oxford et Portland, Oregon, Hart Publishing, 2015.
Rony Brauman, Rony et Eyal Sivan, Un spécialiste, portrait d'un criminel

- Robert Young, Eichmann, Royaume-Uni/Hongrie, 2007.

- Henri Verneuil, I... comme Icare, France, 1979.

- Margarethe von Trotta, Hannah Arendt, Allemagne/France, 2013 